of entry can be established with a combination of computed tomographic scanning, echocardiographic analysis, aortographic analysis, and digital palpation. Use of epiaortic scanning can be cumbersome through a small incision. In patients with COPD, chest wall integrity provided by a partial sternotomy sustains efficient ventilation when combined with effective postoperative analgesia. Absolute contraindications for the TAo approach are "porcelain" aorta and previous sternal wound infection with chest wall reconstruction.

The TF approach is associated with $11 \%$ major vascular complications, ${ }^{1}$ and the TA approach is associated with site-specific complications, such as left ventricular bleeding, hypokinesia/akinesia of the apex, and aneurysm formation. ${ }^{2}$ Stroke remains a significant problem after TAVI compared with SAVR $(5.1 \%$ vs $2.4 \%) .{ }^{1,2}$ Various mechanisms of stroke during TAVI include crossing the AV with guide wires, instrumentation of arch atheroma, balloon valvuloplasty, pigtail catheter thrombosis, valve deployment, and postdilatation balloon valvuloplasty. The
TAx approach does not eliminate crossing the aortic arch or its instrumentation, and the approach to the $\mathrm{AV}$ is at an angle that can be cumbersome. The TAo approach minimizes instrumentation of arch atheroma and might reduce the risk of stroke. The TAo approach provides a safe and feasible technique through a familiar route for surgeons.

\section{References}

1. Smith CR, Leon MB, Mack MJ, Miller DC, Moses JW, Svensson LG, et al. Transcatheter versus surgical aortic-valve replacement in high-risk patients. $N$ Engl J Med. 2011;364:2187-98

2. Walther T, Kempfert J, Rastan A, Borger MA, Linke A, Ender J, et al. Transapical aortic valve implantation at 3 years. J Thorac Cardiovasc Surg. 2011 [Epub ahead of print].

3. Bapat V, Khawaja MZ, Attia R, Narayana A, Wilson K, Macgillivray K, et al Transaortic transcatheter aortic valve implantation using Edwards Sapien valve: a novel approach. Catheter Cardiovasc Interv. 2011 [Epub ahead of print]

4. Jahangiri M, Laborde JC, Roy D, Williams F, Abdulkareem N, Brecker S. Outcome of patients with aortic stenosis referred to a multidisciplinary meeting for transcatheter valve. Ann Thorac Surg. 2011;91:411-5.

5. Tabata M, Umakanthan R, Cohn LH, Bolman RM III, Shekar PS, Chen FY, et al. Early and late outcomes of 1000 minimally invasive aortic valve operations. Eur J Cardiothorac Surg. 2008;33:537-41.

\title{
Carotid artery and jugular vein cannulation in aortic redo surgery
}

\author{
Paul P. Urbanski, MD, PhD, Bad Neustadt, Germany
}

Aneurysms that develop after previous aortic surgery pose a high risk of massive hemorrhage during reopening of the thorax, and therefore a femorofemoral cannulation and deep hypothermia are usually recommended before sternotomy. ${ }^{1,2}$ Here, an alternative approach with arterial cannulation of the common carotid artery through a vascular side graft and venous cannulation of the jugular vein is presented.

From the Cardiovascular Clinic Bad Neustadt, Bad Neustadt, Germany.

Disclosures: Dr Urbanski discloses a financial relationship with MAQUET Cardiovascular, Inc.

Received for publication July 1, 2011; accepted for publication Oct 31, 2011; available ahead of print Dec 12, 2011.

Address for reprints: Paul P. Urbanski, MD, PhD, Herz- und Gefaess-Klinik, Salzburger Leite 1, 97616 Bad Neustadt, Germany (E-mail: p.urbanski@herzchirurgie.de). J Thorac Cardiovasc Surg 2012;143:1227-9

$0022-5223 / \$ 36.00$

Copyright (c) 2012 by The American Association for Thoracic Surgery doi:10.1016/j.jtcvs.2011.10.083

\section{CLINICAL SUMMARY}

For the approach to the right common carotid artery and the right jugular vein, an incision was made on the neck along the margin of the sternocleidomastoid muscle before the median sternotomy. The neck vessels were isolated, and after heparinization, the common carotid artery was crossclamped, a longitudinal incision was made, and an 8- or 10-mm vascular prosthesis (InterGard; InterVascular, MAQUET Cardiovascular, La Ciotat, France) was anastomosed to the artery with a continuous 5-0 Prolene suture, as described in detail previously. ${ }^{3,4}$ The jugular vein was cannulated by using the Seldinger technique. For this purpose, intraoperative transesophageal echocardiographic analysis was performed in all patients to ensure echocardiographic guidance for correct positioning of the venous cannula. First, the guide was introduced into the inferior vena cava under transesophageal echocardiographic guidance, and then the femoral venous cannula (Edwards Lifesciences LLC, Irvine, Calif) was placed (Video 1). In one case with additional mitral valve surgery, we used a dual-stage cannula (Estech, San Ramon, Calif) for cannulation.

The resternotomy was not performed until establishment of cardiopulmonary bypass, achievement of mild hypothermia, and decrease in blood pressure to about $60 \mathrm{~mm} \mathrm{Hg}$. 

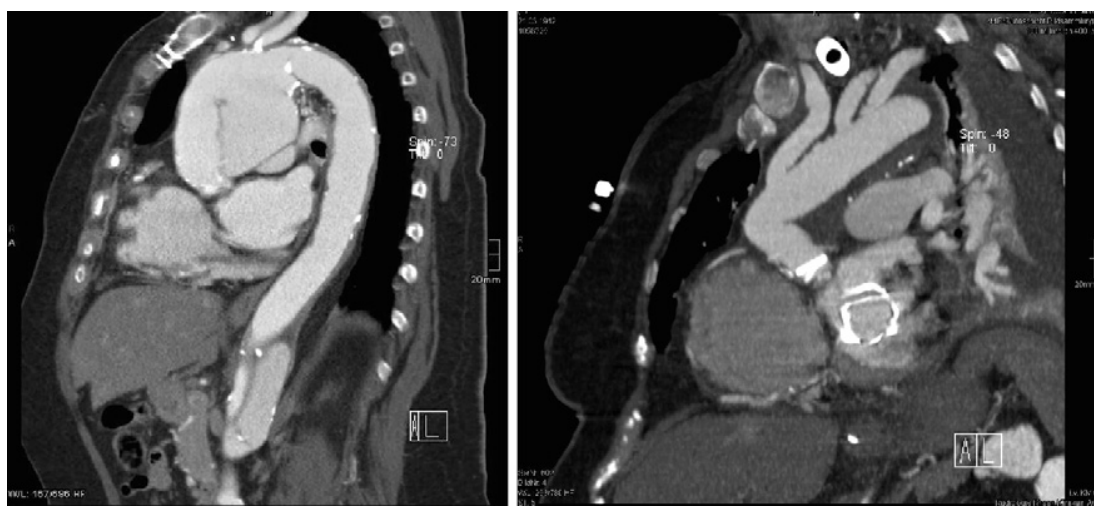

FIGURE 1. Left, Preoperative computed tomographic angiogram showing a huge false aortic aneurysm after ascending aortic replacement caused by acute type A dissection (1992) and aortic valve replacement caused by recurrent aortic insufficiency (2000). Right, Postoperative computed tomographic angiogram after complete arch replacement and complete aortic root replacement with valve composite graft.

Between July 2009 and February 2011, this cannulation technique was performed in 5 consecutive patients. All patients had previous aortic operations, 2 of them twice. The indication for surgical intervention was aortic aneurysm after previous ascending aortic replacement caused by acute type A dissection in 2 cases (Figure 1) and a false aneurysm caused by an aortic prosthesis infection in 3 cases (Figure 2). Cardiopulmonary bypass with flow rates of between 4.3 and $5.6 \mathrm{~L} / \mathrm{min}\left(2.2-2.4 \mathrm{~L} \cdot \mathrm{min}^{-1} \cdot \mathrm{m}^{-2}\right.$ body surface) was sufficient in all cases. Circulatory arrest with a median duration of 35 minutes (range, 9-58 minutes) was used in 3 patients; however, the cerebral perfusion was not interrupted at any time during the operation. The unilateral cerebral perfusion was constantly maintained through the arterial line placed in the carotid artery at a blood temperature of $28^{\circ} \mathrm{C}$ according to our protocol published previously, ${ }^{5}$ regardless of whether circulatory arrest was performed to control the bleeding or to replace the arch. The median cardiopulmonary bypass time, crossclamping time, and rectal temperature were 254 minutes (range, 121-307 minutes), 147 minutes (range, 56-168 minutes), and $28.9^{\circ} \mathrm{C}$ (range, $28.4^{\circ} \mathrm{C}-30.0^{\circ} \mathrm{C}$ ), respectively. The operations consisted of rereplacement of the ascending aorta, complete aortic root, or both (4 patients), or aortic root reconstruction (1 patient) completed by means of total arch replacement, coronary artery bypass grafting, and mitral valve replacement in 2 patients, 2 patients, and 1 patient, respectively. All patients were discharged from the hospital and were alive during the median follow-up time of 10 months (range, 4-24 months).

\section{DISCUSSION}

Use of femorofemoral cannulation for cardiopulmonary bypass is associated with a need for interruption of cerebral perfusion during circulatory arrest, and therefore deep hypothermia is necessary, even if antegrade cerebral perfusion is established by means of additional direct cannulation of the arch arteries after initial complete brain ischemia. Apart from the general negative side effects of deep hypothermia, such as coagulopathy or organ dysfunction, there is also
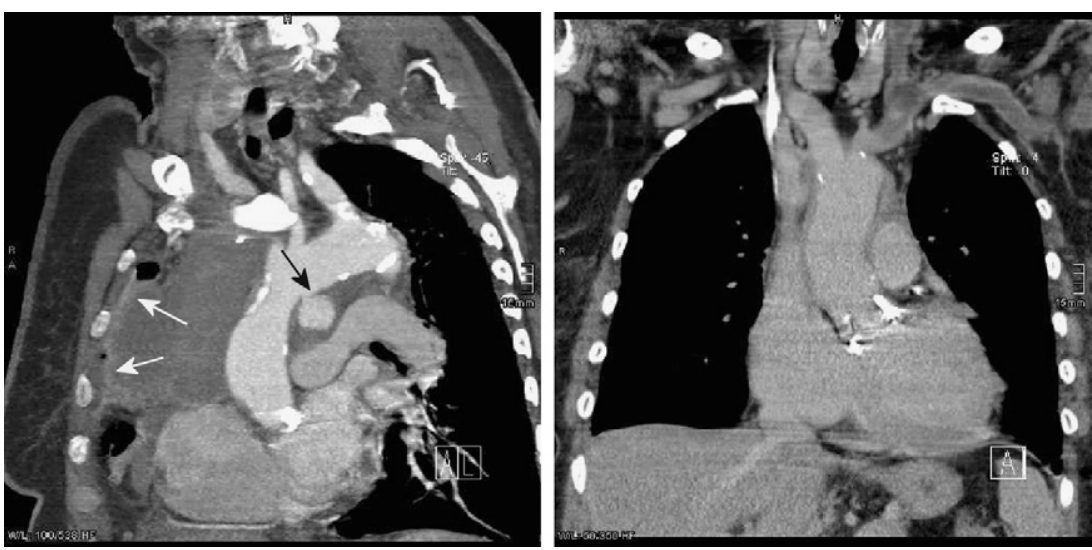

FIGURE 2. Left, Preoperative computed tomographic angiogram showing a huge false aortic aneurysm caused by infection of the vascular prosthesis after ascending aortic replacement. Extension of the false aneurysm to the sternum is marked with white arrows. Leakage of contrast medium at the distal anastomosis is marked with a black arrow. Right, Postoperative computed tomographic angiogram after ascending and hemiarch aortic replacement. 
a particular risk of left ventricular dilatation when the aortic valve is insufficient (this is common in aortic redo operations) and a hypothermic ventricular fibrillation occurs before opening the thorax. In addition, local wound complications are not an exception when a surgical approach to the vessels in the groin is performed.

The approach to the vessels in the neck is, in turn, very fast and easy, even in very obese patients, and local wound complications are extremely rare. ${ }^{3,4}$ Most importantly, when the common carotid artery and jugular vein are cannulated, the cerebral perfusion is constantly maintained during circulatory arrest, eliminating the necessity of deep hypothermia and its negative side effects. Therefore this technique is very suitable in redo operations of the ascending aorta and aortic arch.
I thank Mrs Melissa Lindner, Mrs Alexandra Metz, and Mrs Bianca Müller for their assistance in preparing this article.

\section{References}

1. Malvindi PG, van Putte BP, Heijmen RH, Schepens MAAM, Morshuis WJ. Reoperations for aortic false aneurysms after cardiac surgery. Ann Thorac Surg. 2010; 90:1437-43.

2. Villavicencio MA, Orszulak TA, Sundt TM III, Daly RC, Dearani JA McGregor CGA, et al. Thoracic aorta false aneurysm: what surgical strategy should be recommended? Ann Thorac Surg. 2006;82:81-9.

3. Urbanski PP, Lenos A, Lindemann Y, Weigang E, Zacher M, Diegeler A. Carotid artery cannulation in aortic surgery. J Thorac Cardiovasc Surg. 2006;132: 1398-403.

4. Urbanski PP, Lenos A, Lindemann Y, Zacher M, Frank S, Diegeler A. Use of a carotid artery for arterial cannulation: side-related differences. Thorac Cardiovasc Surg. 2010;58:276-9.

5. Urbanski PP, Lenos A, Bougioukakis P, Neophytou I, Zacher M, Diegeler A. Mildto-moderate hypothermia in aortic arch surgery using circulatory arrest: a change of paradigm? Eur J Cardiovasc Surg. 2011 [Epub ahead of print] 